NOTA DE PESQUISA

\title{
PRIMEIRO RELATO DE Amblyomma fuscum NEUMANN, 1907 (ACARI: IXODIDAE) PARASITANDO LAGARTO DA ESPÉCIE Tupinambis teguixin (L.), NO MUNICÍPIO DE GLORINHA, ESTADO DO RIO GRANDE DO SUL, BRASIL
}

\author{
JOÃO R. MARTINS ${ }^{1}$; ELIDA C. MONTICELLI' ${ }^{1}$; VALÉRIA C. ONOFRIO²; DARCY M. \\ BARROS-BATTESTI ${ }^{2}$; ROVAINA L. DOYLE ${ }^{1}$
}

\begin{abstract}
MARTINS, J.R.; MONTICELLI, E.C.; ONOFRIO, V.C.; BARROS-BATTESTI, D.M.; DOYLE R.L.[The first report of Amblyomma fuscum Neumann, 1907 (Acari Ixodidae) on the lizard Tupinambis teguixin (L.) at the Municipality of Glorinha, State of Rio Grande do Sul, Brazil.] Primeiro relato de Amblyomma fuscum Neumann, 1907 (Acari Ixodidae) parasitando lagarto da espécie Tupinambis teguixin (L.), no município de Glorinha, Estado do Rio Grande do Sul, Brasil. Revista Brasileira de Parasitologia Veterinária, v. 16, n. 4, p. 246-247, 2007. Instituto de Pesquisas Veteri-nárias Desidério Finamor, FEPAGRO, Estrada do Conde, 6000, Eldorado do Sul, RS 92990-000, Brasil. joaorsm@terra.com.br

Amblyomma fuscum known only from Brazil has been described as a rare tick species with few reports of its occurrence in South and Southeast region. This is a new records this tick species (9 females) parasitizing lizard (Tupinambis teguixin) at the Municipality of Glorinha, State of Rio Grande do Sul. The females were deposited in the tick collection of Veterinary Research Institute Desiderio Finamor (7 specimens), Eldorado do Sul, RS and in the Acari collection from Instituto Butantan, São Paulo, State of São Paulo (2 specimens). The finding confirms establishment de A. fuscum in the South of Brazil.
\end{abstract}

KEY WORDS: Amblyomma fuscum, tick, lizard, Rio Grande do Sul.

\section{RESUMO}

Amblyomma fuscum conhecida somente no Brasil, tem sido descrita como uma espécie rara de carrapato com relatos de sua ocorrência nas regiões sul e sudeste. Este é um novo registro desta espécie (9 fêmeas) parasitando lagarto (Tupinambis teguixin), no Município de Glorinha, Estado do Rio Grande do Sul. As fêmeas foram depositadas na coleção do Instituto de Pesquisas Veterinárias Desidério Finamor (7 espécimes) e na coleção de Acari do Instituto Butantan, Estado de São Paulo (2 espécimes). O achado confirma o estabelecimento de A. fuscum no Sul do Brasil.

PALAVRAS-CHAVE: Amblyomma fuscum, carrapato, lagarto, Rio Grande do Sul.

\footnotetext{
${ }^{1}$ Instituto de Pesquisas Veterinárias Desidério Finamor, FEPAGRO, Estrada do Conde, 6000, Eldorado do Sul, RS 92990-000. joaorsm@terra.com.br

${ }^{2}$ Instituto Butantan, Av. Vital Brasil, 1500, São Paulo, SP 05503-900.
}

A ocorrência de Amblyomma fuscum Neumann, 1907 (Acari: Ixodidae) no Estado do Rio Grande do Sul foi primeiramente registrada por Aragão (1936) e recentemente confirmada por Barros-Battesti et al. (2005). O macho desta espécie foi descrito por Neumann (1907), de um lote de cinco exemplares coletados em serpente Boa constrictor L. da América do Sul, sem registro de localidade, ou qualquer outra informação. Aragão (1936) descreveu a fêmea que foi encontrada em lagarto na localidade de Rio Velha, RS juntamente com mais 3 machos. $\mathrm{O}$ autor mencionou ainda outro material de $A$. fuscum coletado na Ilha do Curral, RS onde relatou o encontro de 4 machos e 2 fêmeas. Evans et al. (2000) comentaram que a espécie A. fuscum, apesar de ter sido ignorada na lista de espécies válidas por Keirans (1992), apareceu na lista elaborada por Camicas et al. (1998). Horak et al. (2002) não mencionaram o táxon, porém, Guglielmone et al. (2003) mantiveram a validade da espécie com base nas informações contidas em coleções brasileiras. Barros-Battesti et al. (2005) após compararem espécimes originários do Brasil depositados na Coleção Acarológica do Instituto Butantan, SP, com o macho 
tipo depositado no museu de História Natural de Leiden, Holanda, redescreveram o macho e a fêmea baseados em microscopia óptica e de varredura. Exceto pelos tipos e pelo material depositado em Rostock (SCHULZE, 1936), ambos de localidade desconhecida, todos os outros relatos conhecidos são do Brasil.

Os espécimes de $A$. fuscum aqui relatados foram encontrados no lagarto Tubinambis teguixin (L.) coletado no município de Glorinha, Estado do Rio Grande do Sul (2955’S; $50^{\circ} 50^{\prime} \mathrm{W}$ ). Foram colhidas 9 fêmeas com idiossoma variando de 5 a $9 \mathrm{~mm}$ de comprimento por 3 a $6 \mathrm{~mm}$ de largura, e escudo mais escuro nas porções laterais onde se concentram as grandes pontuações típicas da espécie, que foi identificada pela chave dicotômica de Onofrio et al. (2006).

Sua ocorrência parece estar restrita aos estados de São Paulo, Santa Catarina e Rio Grande do Sul, a não ser por um relato para Estado de Pernambuco, que não pode ser confirmado (CUNHA et al., 1999; BARROS-BATTESTI et al, 2005; MARQUES et al., 2006). Répteis têm sido descritos como hospedeiros preferenciais para o estágio adulto de $A$. fuscum (GUIMARÃES et al., 2001; GUGLIEMONE et al., 2003; BARROS-BATTESTI et al., 2005). Há um relato em anfíbio da espécie Chaunus arenarum (Hensel) citado por Sinkoc e Brum (1997) como Bufo arenalis, cuja nomenclatura foi recentemente atualizada por Frost et al. (2006), e em mamíferos Cerdocyon thous (L.), Canis familiaris (L.) e Dasypus septemcinctus (L.) (BRUM et al., 2003; BARROSBATTESTI et al., 2005). O único registro para imaturo de A. fuscum é de uma ninfa em Didelphis aurita (WiedNeuwied) (BARROS-BATTESTI et al., 2005). Apesar dos poucos relatos e do estágio adulto ter sido associado aos répteis, os registros em mamíferos, incluindo humanos (MARQUES et al., 2006), parecem indicar uma baixa especificidade parasitária para esta espécie.

Agradecimentos:- Aos pesquisadores Carlos Jared e Marta Antoniazzi, ambos do Laboratório de Biologia Celular do Instituto Butantan, pela atualização da nomenclatura de anfíbios.

\section{REFERÊNCIAS BIBLIOGRÁFICAS}

ARAGÃO, H. B. Ixodidas brasileiros e de alguns paizes limitrophes. Memórias do Instituto Oswaldo Cruz, v.31, n.4, p. 749-843, 1936.

BARROS-BATTESTI, D.M.; ONOFRIO,V.C.; LABRUNA, M.B.; MARTINS, J.R.; GUGLIELMONE, A.A. Redescription of Amblyomma fuscum Neumann, 1907 (Acari:Ixodidae), a rare tick in South America Brazil. Systematic Parasitology, v.61, n.2, p. 85-92, 2005.

BRUM, J.G.W.; VALENTE, A.L.S. ; ALBANO, A.P.; COIMBRA, M.A.C.; GREQUE, G.G. Ixodidae de mamíferos silvestres atendidos no Núcleo de Reabilitação da Fauna Silvestre, UFPEL. Arquivos do Instituto Biológico, v.70, n.2, p. 211-212, 2003.
CAMICAS, J.L.; HERVY, J.P.; ADAM, F.; MOREL, P.C. Les tiques du monde. Paris: Ed.Orstom, Institut Français de Recherche Scientifique pour le Développement en Coopération, 1998. 223 p.

CUNHA, M.C.A.L.; FARIAS, A.M.I.; BRITO, F.L.; SERRAFREIRE, N.M. Ocorrência de Amblyomma fuscum Neumann, 1907 (Acari:Ixodidae) em Boa constrictor Linnaeus, 1758 (Reptilia: Boidae) no estado de Pernambuco, Brasil. Entomologia y Vectores, v.6, n.5, p. 577-579, 1999.

EVANS, D.E.; MARTINS, J.R.; GUGLIELMONE, A.A. A review of the ticks (Acari: Ixodida) of Brazil, their hosts and geographical distribution. 1. The state of Rio Grande do Sul, southern Brazil. Memórias do Instituto Oswaldo Cruz, v.95, n.4, p. 453-470, 2000.

FROST, D.R.; GRANT, T.; FAIVOVICH, J.; BAIN, R.H.; HAAS, A.; HADDAD, C.F.B.; DE SÁ, R.O.; CHANNING, A.; WILKINSON, M.; DONNELLAN, S.C.; RAXWORTHY, C.J.; CAMPBELL, J.A.; BLOTTO, B.L.; MOLER, P.; DREWES, R.C.; NUSSBAUM, R.A.; LYNCH, J.D.; GREEN, D.M.; WHEELER, W.C. The amphibian tree of life. Bulletin of the American Museum of Natural History, n. 297: p. 1-370, 2006.

GUGLIELMONE, A.A.; ESTRADA-PEÑA, A.; KERIANS, J.E.; ROBBINS, R.G. Ticks (Acari: Ixodida) of the Neotropical Zoogeographic Region. Houten: International Consortium on Ticks and Tick-borne Diseases (ICTTD2), 2003. 173p.

GUIMARÃES, J.H.; TUCCI, E.D.; BARROS BATTESTI, D.M. Ectoparasitos de importância veterinária. São Paulo: Pleiade-FAPESP, 2001. 213 p.

HORAK, I.G.; CAMICAS, J.L.; KEIRANS, J.E. The Argasidae, Ixodidae and Nuttalliellidae (Acari: Ixodida): a world list of valid tick names. Experimental and Applied Acarology, v. 28, n. 1, p. 27-54, 2002.

KEIRANS, J.E. Systematics of the ixodida (Argasidae, Ixodidae, Nuttaliellidae): an overview and some problems. In: FIVAZ, B.H., PETNEY, T.N., HORAK, I.G. (Eds.) Tick vector biology medical and veterinary aspects. Berlin: Springer Verlag, 1992. p. 1-21.

MARQUES, S.; COL, R.D.; JÚNIOR, M.O.M. ; GONÇALVES, E.F.B.; PINTER, A.; LABRUNA, M.B. Parasitismo de Amblyomma fuscum (Acari:Ixodidae) em humanos. Ciência Rural, v. 36, n. 4, p.1328-1330, 2006.

NEUMANN, L.G. Quatre espèces nouvelles d'ixodidés. Notes from the Leyden Museum, v. 29, n. 2, p. 88-100, 1907.

SINKOC, A.L.; BRUM, J.G.W. Ocorrência de Amblyomma fuscum Neumannm 1899 e Amblyomma humerale Koch, 1844 (Acari: Ixodidae) em Bufo arenalis no estado de São Paulo, Brasil. Ciência Rural, v.27, n.2, p. 339-340, 1997.

SCHULZE, P. Neue und wenig bekannte Amblyommen und Aponommen aus Afrika, Südamerika, Indien, Borneo und Australien. (Ixodoidea). Zeitschrift für Parasitenkunde, v. 8, p. 691-637, 1936.

Recebido em 12 de junho de 2007.

Aceito para publicação em 12 de dezembro de 2007. 